\title{
Efficient data assimilation into a complex, 3-D physical-biogeochemical model using partially-local Kalman filters
}

\author{
I. Hoteit ${ }^{1}$, G. Triantafyllou ${ }^{2}$, and G. Petihakis ${ }^{2}$ \\ ${ }^{1}$ Scripps Institution of Oceanography, La Jolla, CA, USA \\ ${ }^{2}$ Hellenic Centre for Marine Research, Anavissos, Greece
}

Received: 1 December 2004 - Revised: 11 August 2005 - Accepted: 9 September 2005 - Published: 30 November 2005

\begin{abstract}
Advanced Kalman filtering techniques were used to assimilate pseudo ocean color and profile data into a complex, three-dimensional coupled physical (POM)biogeochemical (ERSEM) model of the Cretan Sea ecosystem. The assimilation schemes, the Singular Evolutive Partially-Local Extended Kalman (SEPLEK) filter and its variant called SFPLEK, are based on the standard SEEK filter in which the Kalman correction is made along a set of "global" and "local" directions, determined via a so-called "global-local EOF analysis". The global functions are used to represent the ecosystem large-scale variability. They are allowed to evolve in time in the SEPLEK filter to follow changes in the model dynamics, while they remain invariant in the SFPLEK filter. The local functions always remain invariant and are determined in such a way as to independently represent the different spatial regimes of the ecological model. This helps to improve the estimation of finescale variations while requiring significantly less computational time compared to the SEEK filter.
\end{abstract}

Several assimilation experiments were performed to assess the relevance of the assimilation system and to study its sensitivity to different choices of global/local EOFs. The SFPLEK filter was used in all the sensitivity experiments in order to efficiently measure the representativeness of the different set of correction directions, as well as to save computational time. Assimilation results suggest that the use of global-local correction directions clearly enhances the filter's performance under different assimilation setups. The choice of the local directions should, however, be carefully considered, taking into account the model regional variability and the characteristics of the observational system.

Keywords. Oceanography: General (numerical modeling; Ocean prediction) - Biological and Chemical (ecosystems and ecology)

Correspondence to: G. Triantafyllou

(gt@ath.hcmr.gr)

\section{Introduction}

The Cretan Sea is located in the northwest part of the eastern Mediterranean Sea. It is linked with the Levantine basin and the Ionian Sea through the eastern and western straits of the Cretan Arc. The area is dominated by multiple scale circulation patterns with a complex hydrological structure characterized by mesoscale variability. As described in a 3-D modeling study of the Cretan Sea (Petihakis et al., 2002) the cyclonic circulation to the north of the central and eastern part of the island, in conjunction with the anticyclonic circulation in the north central part, results in areas of increased production around the cyclone, in contrast to the area affected by the anticyclonic activity. The system is characterized by a seasonal thermocline $(60-80 \mathrm{~m})$ from spring to autumn, separating the water column into two layers. Primary and bacterial production decrease moving offshore while increased production rates are associated with intense mixing events and the subsequent nutrient intrusion to the euphotic zone. Thus most of the primary production in the euphotic zone is supported by regenerated nutrients. As in all complex systems, advanced numerical models seem to be the only option for the simulation of the behavior and the evolution of the Cretan Sea ecosystem (see Thingstad et al., 1999). Such models, however, include a significant number of parameters which are usually provided by the scientific literature, having been derived through laboratory experiments and in-situ observations of a similar type ecosystem. To advance beyond this subjective approach, data assimilation is the process of finding the model solution which is most consistent with the observations. These techniques are essential in producing the most complete picture of the system by integrating all available knowledges, including observations (in-situ and satellite data) and biophysical principles (state-of-the-art numerical models).

Data assimilation methods can be classified into two categories: variational methods based on the optimal control theory and sequential methods based on the statistical estimation theory (see Malanotte-Rizzoli, 1996). Variational methods 
have been considered by a number of studies (Evans, 1999; Matear, 1995; Harmon and Challenor, 1996). For instance, Lawson et al. (1996) used the well-known adjoint method with a simple ecosystem model. The same method has recently been efficiently implemented by Faugeras et al. (2003) to assimilate chlorophyll data into a 1-D ecosystem model. Relying on the assumption that a coupled model with a given set of parameters can reproduce the observations, variational methods can be strongly sensitive to the unresolved processes of the model formulation. Although model error can be in principle, considered by applying a weak constraint inverse formulation (Natvik et al., 2001), this would make the dimension of the search so long that unpreconditioned searches for a best fit would be prohibitive. Sequential methods which allow one to incorporate the model error seem, therefore, to be more appropriate for data assimilation into marine ecosystem models.

The application of sequential methods for data assimilation into ecosystem models has received more attention during the last decade. These techniques are generally derived from the extended Kalman (EK) filter. Indeed, the implementation of the EK filter in realistic ocean models is not possible because of its prohibitive computational burden. Different simplified forms of this filter have therefore been developed, basically reducing the dimension of the system through some kind of projection onto a low dimensional subspace (Cane et al., 1995; Fukumori and Malanotte-Rizzoli, 1995; Verlaan and Heemink, 1995). Recently, Monte Carlo techniques were also introduced to avoid the linearization of the model equations, leading to the ensemble Kalman methods (Evensen and van Leeuwen, 1994; Burgers et al., 1998). Allen et al. (2003) and Natvik and Evensen (2003) demonstrated the effectiveness of the ensemble Kalman filter for data assimilation with a 1-D, three-component ecosystem model.

The singular evolutive extended Kalman (SEEK) filter is a simplified square-root EK filter which has been introduced by Pham et al. (1998). It basically makes use of singular, low-rank matrices approximations of the filter's error covariance matrix. This leads to the application of the Kalman's correction only along the directions for which the forecast error was not sufficiently attenuated by the system. The SEEK filter has been successfully implemented in several marine ecosystem models. Carmillet et al. (2001) assimilated ocean color data with a 3-D biophysical model of the north Atlantic Ocean, while keeping the correction directions invariant in time to reduce computational time (called hereafter the Singular fixed extended Kalman (SFEK) filter). The same filter has also been used by Hoteit et al. (2003) to assimilate real Oxygen and Nitrate data into a 1-D ecosystem model. The behavior of this filter can, however, be degraded in the presence of model instabilities, generally not well represented by an invariant set of correction directions (Hoteit et al., 2002). A further extension of the 1-D to a 3-D system has been presented by Triantafyllou et al. (2003a) with the first application of an advanced Kalman filtering technique (ensemble variant of the SEEK filter, called SEIK filter) for data assimilation into a complex, three-dimensional marine ecosystem model. In the latter work, however, the number of directions was limited by the available computational resources.

Limits in computer capacity further complicate the estimation of short-range phenomena with Kalman filtering techniques, generally inefficient when dealing with such intermittent processes (Bennett, 1992). This makes the data assimilation into marine ecosystem models particularly difficult because an important part of the variability of these models is governed by fine-scale variations. In this study, the SemiEvolutive Partially-Local Extended Kalman (SEPLEK) was used to tackle these problems. This filter was found to be more performant than the SEEK filter when dealing with variables having short-range variability, while requiring significantly less computational time (Hoteit et al., 2001). This is possible due to the ability of adapting the correction directions of this filter to the different spatial regimes of the system under study. Specifically, global and local functions are used to better represent the long-range and the different short-range variability of the model, using a so-called "global-local" Empirical Orthogonal Functions (EOF) analysis. Only the global functions are allowed to evolve in time to follow changes in the model dynamics, while the local functions remain invariant. This significantly reduces the computational burden compared to the SEEK filter, as the number of required global functions is generally small. Additional cost reduction can be further obtained by keeping the global functions invariant, which also provides the best way to assess the representativeness of a given set of correction directions (Brasseur et al., 1999). This simplified variant of the SEPLEK filter was, therefore, used in our sensitivity experiments. It has the same algorithm as the SFEK filter, but makes use of partially local correction directions. Hence, it will be called the Singular Fixed Partially Local Extended Kalman (SFPLEK) filter, in order to remove any confusion with the (classical) SFEK filter which was also considered in this study. Note that, although this study is closely related to that of Triantafyllou et al. (2003a) mentioned above, since the same ecosystem model was used in both studies, the results of this work were not linked to the latter, in order to avoid a complex comparison between an ensemble filter and a partially-local extended filter. It is actually more appropriate to carry out this comparison using a local variant of the SEIK filter, which can be developed in a similar way to the local Ensemble Kalman filter (Ott et al., 2004). This will be one of our future objectives. In this study, however, we decided to only focus on the evaluation of the benefits of using local EOFs in an ecological assimilation system.

Numerical experiments were performed to assess the relevance of the assimilation system and to study its sensitivity to different parameters and setups. An important part of this work is devoted to studying the impact of the choice of local EOFs on the performance of the filter. For instance, Carmillet et al. (2001) already tested the idea of separating the euphotic zone from the deep ocean by excluding the deep ocean variables from the state vector of the filter. This idea is further extended here while representing these two different regimes, using independent (vertical) local functions instead 


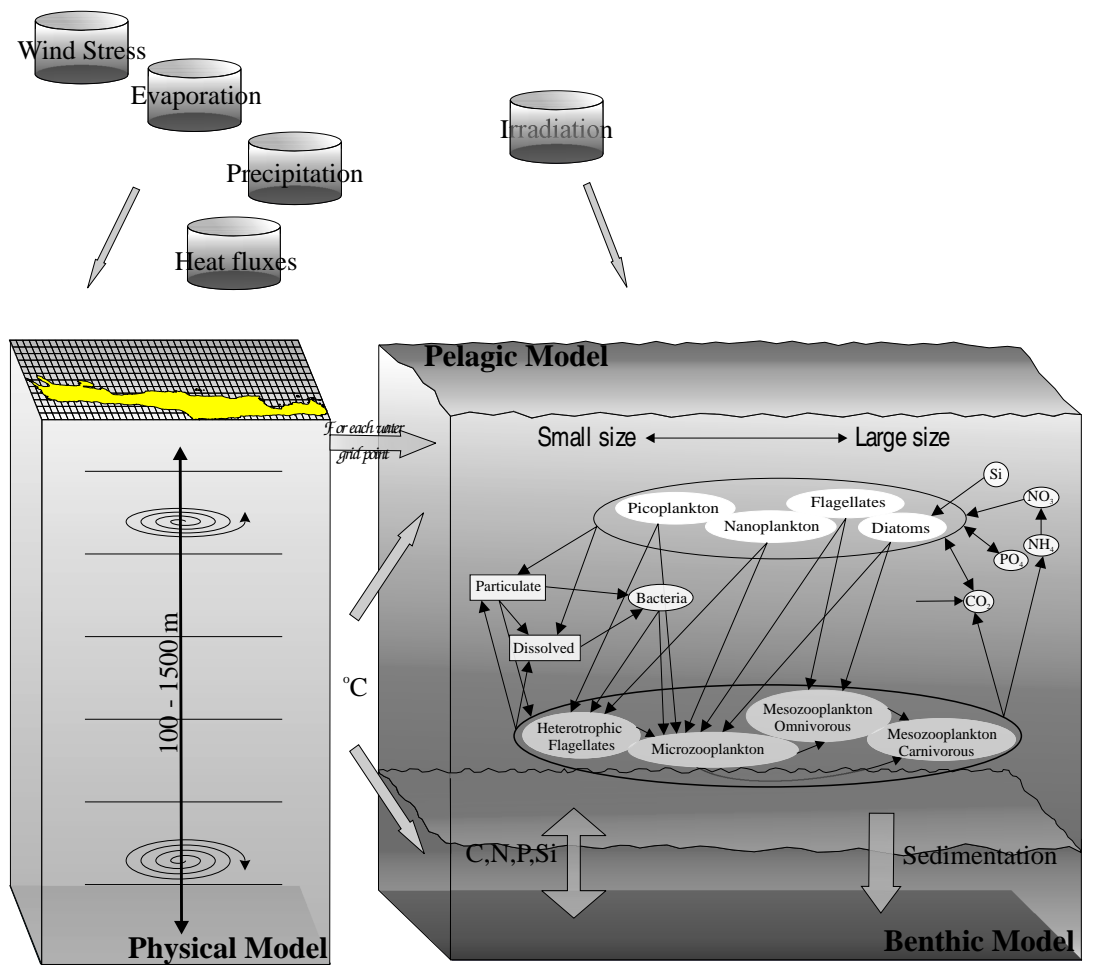

Fig. 1. Schematic representation of the ecosystem food web.

of neglecting the corrections in the deep ocean. Several horizontal decompositions are also considered to separate the different spatial regimes in the model. We also show how the choice of the local EOFs should take into account the characteristics of the data to be assimilated by limiting the filter's corrections to the areas where the data are available. The paper is organized as follows. In Sect. 2 the coupled biophysical model is described. Section 3 summarizes the basic elements of the SEPLEK filter. The setup of the assimilation experiments is presented in Sect. 4. The assimilation results are illustrated and discussed in Sect. 5, while the summary and conclusions are given in Sect. 6.

\section{The ecosystem model}

The biophysical model used in this study emanates from the coupling of the physical Princeton Ocean Model (POM) (Blumberg and Mellor, 1987), a primitive equation model and the generic European Regional Seas Ecosystem Model (ERSEM) (Baretta et al., 1995), which describes the biogeochemical processes. Both sub models have been customed tuned and validated for the Cretan Sea, as described in detail in Petihakis et al. (2002) and Triantafyllou et al. (2003a), producing a rather efficient representation of both physical and biological components. Although, as mentioned before, both models have been already described elsewhere, a brief reference on the key features is considered to be useful for the readership.
POM is a three-dimensional, time dependent ocean model, including a higher order turbulence closure scheme (Blumberg and Mellor, 1987), with equations being solved through the use of an Arakawa-C differencing scheme. A sigma coordinates discretization is adopted for the vertical grid dimension, while for the time integration, a split explicit scheme is applied, with the barotropic and baroclinic modes being integrated separately using a leap frog scheme with different time steps.

Although one of the biggest advantages of ERSEM is its generic nature, significant modifications were necessary prior to its application to the oligotrophic Cretan Sea ecosystem, as shown in Fig. 1 (Petihakis et al., 2002; Triantafyllou et al., 2001, 2003b). The use of a functional group idea where organisms with similar processes are grouped together, rather than that of a species, increases its portability to any type of system. This, however, requires a good and sound knowledge of the role of each biological component by the user. The biotic system encompasses the three major types (producers, consumers and decomposers), with each type being further subdivided, thus increasing the required complexity into 88 variables. The backbone of the model is the carbon dynamics, with dynamically variable ratios of the N:P:Si elements. Although ERSEM offers a full benthic system module, in this study, due to the increased computer cost, the simple first order benthic returns module was used.

The modeled area is plotted in Fig. 2, extending from $23.575^{\circ} \mathrm{E}$ to $26.3^{\circ} \mathrm{E}$ and $35.075^{\circ} \mathrm{N}$ to $36^{\circ} \mathrm{N}$. A horizontal resolution of 6 minutes was chosen, thus producing $56 \times 20$ 


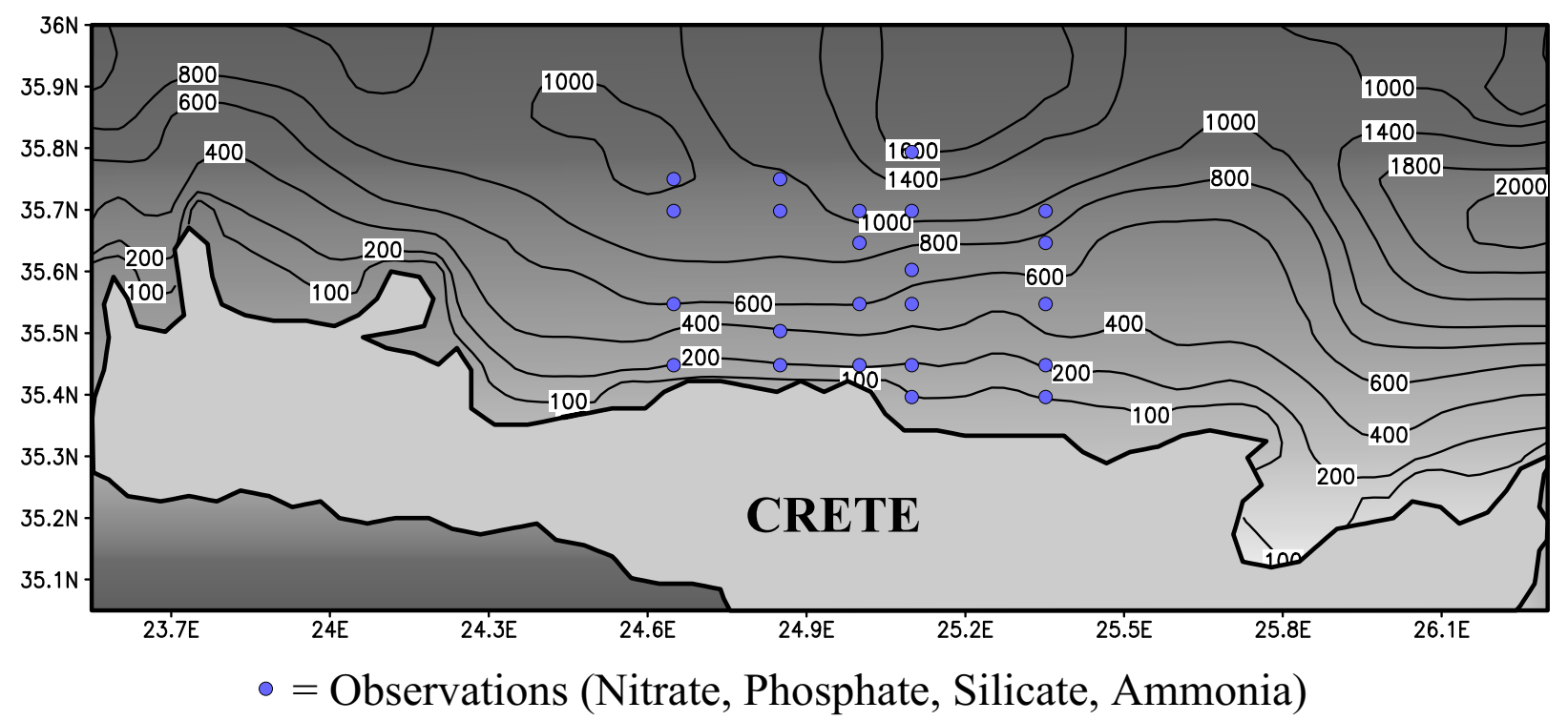

Fig. 2. Simulation domain with locations for profiles (pseudo) observations of nitrate, phosphate, silicate and ammonia.

elements. In an attempt to account for the increased velocity gradients near the surface, a logarithmic vertical distribution was used with a total of 30 layers. The model was initialized with climatological objectively analyzed temperature and salinity profiles from the Mediterranean Ocean Database (MODB-MED4), with a resolution of $1 / 4^{\circ} \times 1 / 4^{\circ}$ for each MODB layer. Additionally, all initial velocities were set to zero. Wind stress fields derived from the ECMWF 19791993 6-h reanalysis data and climatological heat flux fields of Bignami et al. (1995) were used for atmospheric forcing, while all biological variables were initialized from the 3-D ecological model for the Cretan Sea (Petihakis et al., 2002).

\section{The SEPLEK filter}

The estimation of long-range correlations associated with remote observations is a well-known difficulty of Kalman filtering methods (Bennett, 1992; Houtekamer and Mitchell, 1998). To tackle this problem, Houtekamer and Mitchell (1998) simply used a cutoff radius to exclude the effects of observations far away from the analysis location. However, the introduction of an "artificial" radius may be inconsistent with the correlations present in the estimation error covariance matrix, and might lead to numerical noise. Recently, a new framework to deal with this problem has been under development. It essentially involves the decomposition of the model domain into several sub-domains and the application of Kalman's correction independently in each subdomain, using an associated estimation covariance matrix (Anderson, 2003). Additional simplifications were also introduced to further reduce the computational burden using coarser grids (Fukumori, 2002) or EOFs (Testut et al., 2003; Ott et al., 2004), within the context of reduced-order and ensemble techniques, respectively. With the same aim in view,
Hoteit et al. (2001) introduced the SEPLEK filter to improve the local behavior of the SEEK filter and to reduce its computational cost. The main idea behind this filter is to force the correction directions of the SEEK filter to be local, by having as a support a small region of the domain, using a so-called "local EOF analysis". To improve the representation of the long-range variability, the local functions were complemented by global (classical) EOFs, leading to a set of "global-local" correction directions. The global functions can further be allowed to evolve with the model dynamics, as in the SEEK filter. The local functions remain invariant in order to retain their locality. This drastically reduces the computational burden with respect to the SEEK filter, since the number of global functions in practice can be small. It is important to note that, although the idea of the SEPLEK filter is similar to the filters cited above, its principle is different. The correction step of the Kalman filter actually remains unchanged in the SEPLEK filter and it is always applied globally, while making use of global and local correction functions.

\subsection{The global-local correction directions}

To improve the representation of short-range correlations, a so-called "local EOF analysis" was introduced by Hoteit et al. (2001). The basic idea is to construct a set of EOFs, each having as a support a small region of the model domain. This is done by applying separate EOF analysis on a set of model sub-domains. To obtain adequate representation, these sub-domains should overlap. One efficient way to do this is to consider a partition of unity of the model domain, i.e. a set of positive functions $\left\{\chi^{(j)}, j=1, \ldots, J\right\}$ defined over the model domain whose sum is identically equal to 1 . The model state vector $X$ can then be decomposed into local fields $X^{(j)}$, such as $X=\sum_{j=1}^{J} X^{(j)}$ with $X^{(j)}=X \chi^{(j)}$. An 
independent (classical) EOF analysis is then performed on each set of local fields $X^{(j)}$ to determine a representational subspace for each sub-domain. The "global" representational error of the original state vector $X$ can still be reduced by considering its projection onto the subspace spanned by the set of (local) EOFs of all the sub-domains.

Since the use of local functions only, may degrade the representation of the long-range variability, Hoteit et al. (2001), proposed to augment the local EOFs with some global functions. This would provide a set of functions suitable for the representation of the global, as well as the local model variability. Such a set of "global-local" functions is obtained by performing a local EOF analysis on the residuals of the vectors $X_{1}, \ldots, X_{N}$ in the first global EOFs. This is supported by the fact that these residuals are generally due to the bad representation of the local variability in the subspace spanned by the global EOFs. Recently, a similar idea was tested by Waseda et al. (2003), but using wavelets to represent the local fine-scale information.

\subsection{Filter's initialization}

The global-local analysis does not readily provide a low-rank approximation of the error covariance matrix needed for the initialization of the filter. Therefore, we resort to an objective analysis to correct the initial state estimate $\bar{X}$ using the initial observations $Y_{0}$. In most applications, $\bar{X}$ is assumed to be the average of a set of state vectors (provided by a historical run).

Starting from a set of global-local EOFs $L_{0}^{B}=\left[L_{0} \vdots B\right]$, with $r_{g}$ global EOFs $\left(L_{0}\right)$ and $r_{l}$ local EOFs $(B)$, we take as initial analysis state (Hoteit et al., 2001)

$X^{a}\left(t_{0}\right)=\bar{X}+L_{0}^{B} U_{0}\left(L_{0}^{B}\right)^{T} \mathbf{H}_{0}^{T} R_{0}^{-1}\left(Y_{0}^{o}-H_{0} \bar{X}\right)$,

where $U_{0}=\left[\left(L_{0}^{B}\right)^{T} \mathbf{H}_{0}^{T} R_{0}^{-1} \mathbf{H}_{0} L_{0}^{B}\right]^{-1}, R_{0}$ is the initial observational error covariance matrix, and $\mathbf{H}_{0}$ is the gradient of $H_{0}$ evaluated at $\bar{X}$. This provides an initial analysis error covariance matrix of rank $\left(r_{g}+r_{l}\right)$,

$P^{a}\left(t_{0}\right)=L_{0}^{B} U_{0}\left(L_{0}^{B}\right)^{T}$.

\subsection{Filter's algorithm}

As the Kalman filter, the SEPLEK filter provides an estimate of the system state as a succession of forecast and correction steps. Consider a physical system

$X^{t}\left(t_{k}\right)=M\left(t_{k-1}, t_{k}\right) X^{t}\left(t_{k-1}\right)+\eta_{k}$,

where $X^{t}(t)$ is the true state vector at time $t, M(s, t)$ is an operator describing the system transition from time $s$ to time $t$, and $\eta_{k}$ represents the model error. The forecast $X^{f}\left(t_{k}\right)$ is obtained by integrating the model forward in time, starting from the most recent analyzed state $X^{a}\left(t_{k-1}\right)$. When a new observation $Y_{k}^{o}$ is made, it is assumed to be a function of $X^{t}$ and a measurement of uncertainty

$Y_{k}^{o}=H_{k} X^{t}\left(t_{k}\right)+\varepsilon_{k}$, where $H_{k}$ is the observational operator and $\varepsilon_{k}$ represents the error in the observations. $\eta_{k}$ and $\varepsilon_{k}$ are assumed to be independent and normally distributed with zero mean and covariance matrix $Q_{k}$ and $R_{k}$, respectively. Assuming that a set of global-local correction directions $L_{k}^{B}$ is available, the SEPLEK filter provides the analysis state, while using the observations sequentially to correct the forecast along the directions of $L_{k}^{B}$ only, hence it will be called the "correction basis" of the filter, exactly as in the SEEK filter (Pham et al., 1998),

$X^{a}\left(t_{k}\right)=X^{f}\left(t_{k}\right)+L_{k}^{B} U_{k}\left(L_{k}^{B}\right)^{T} \mathbf{H}_{k}^{T} R_{k}^{-1}\left[Y_{k}^{o}-H_{k} X^{f}\left(t_{k}\right)\right]$,

where $\mathbf{H}_{k}$ is the gradient of $H_{k}$ evaluated at $X^{f}\left(t_{k}\right)$ and $U_{k}$ is updated with

$U_{k}^{-1}=\left[U_{k-1}+P_{L_{k}^{B}}^{T} Q_{k} P_{L_{k}^{B}}\right]^{-1}+\left(L_{k}^{B}\right)^{T} \mathbf{H}_{k}^{T} R_{k}^{-1} \mathbf{H}_{k} L_{k}^{B}$.

In the above equation, $P_{L_{k}^{B}}=\left[\left(L_{k}^{B}\right)^{T} L_{k}^{B}\right]^{-1}\left(L_{k}^{B}\right)$ is the projection operator onto the subspace generated by $L_{k}^{B}$. The corresponding analysis error covariance matrix is then given by

$P^{a}\left(t_{k}\right)=L_{k}^{B} U_{k}\left(L_{k}^{B}\right)^{T}$.

To attenuate the bad impact of different approximations and uncertainties on the performance of the filter, a forgetting factor $\rho$ is used here. This factor enhances the filter stability by amplifying the already existing modes of the background error. It simply amounts to replacing Eq. (6) by

$U_{k}^{-1}=\left[1 / \rho U_{k-1}+P_{L_{k}^{B}}^{T} Q_{k} P_{L_{k}^{B}}\right]^{-1}+\left(L_{k}^{B}\right)^{T} \mathbf{H}_{k}^{T} R_{k}^{-1} \mathbf{H}_{k} L_{k}^{B}$.

\subsection{Evolution of the correction directions}

The evolution of the correction directions is generally beneficial since it allows the filter to keep track of changes in the model dynamics (Hoteit et al., 2002). When a set of globallocal functions is used as a correction basis for the filter, only the global functions can be allowed to evolve with the model dynamics, as in the SEEK filter, since the local EOFs would lose their local character. Although this significantly reduces the computational burden of the method, the "non-evolutive" nature of the local functions is clearly a disadvantage within the framework of the SEEK filter. This approximation, however, is not expected to place a major limitation on the filter's performance, since the evolution equation of the SEEK filter is designed to follow only slow changes (Pham et al., 1998). This helps to support the idea of keeping the local functions invariant, since their evolution might be sometimes problematic as they represent quickly evolving local variations by design. This means that we aim at not correcting all fine-scale variation errors, but only a large number of them, on average. If the correction basis is already decomposed as $L_{k-1}^{B}=\left[L_{k-1} \vdots B\right]$ at time $t_{k-1}$, we therefore take

$L_{k}^{B}=\left[L_{k} \vdots B\right]$, 
Table 1. Twin experiments setup.

\begin{tabular}{ll}
\hline Assimilation Scheme & SFEK, SFPLEK, SEPLEK \\
State vector & Ecosystem state variables \\
Dimension of state vector & 1768536 \\
EOF analysis & $\approx 3$ years (360 vectors) \\
Assimilation Period & 5 March-5 June (3 months) \\
Observed Variables & Sea color or Profiles of \\
& Nitrate, Phosphate, Silicate, \\
& Ammonia \\
Number of Observations & Sea color: 693 \\
& Profiles: 23 stations $\times 30$ layers \\
& $=690$ \\
Assimilation time step & 4 days \\
Observation Error & Sea color: $10 \%$ \\
& Profiles: $5 \%$ \\
\hline
\end{tabular}

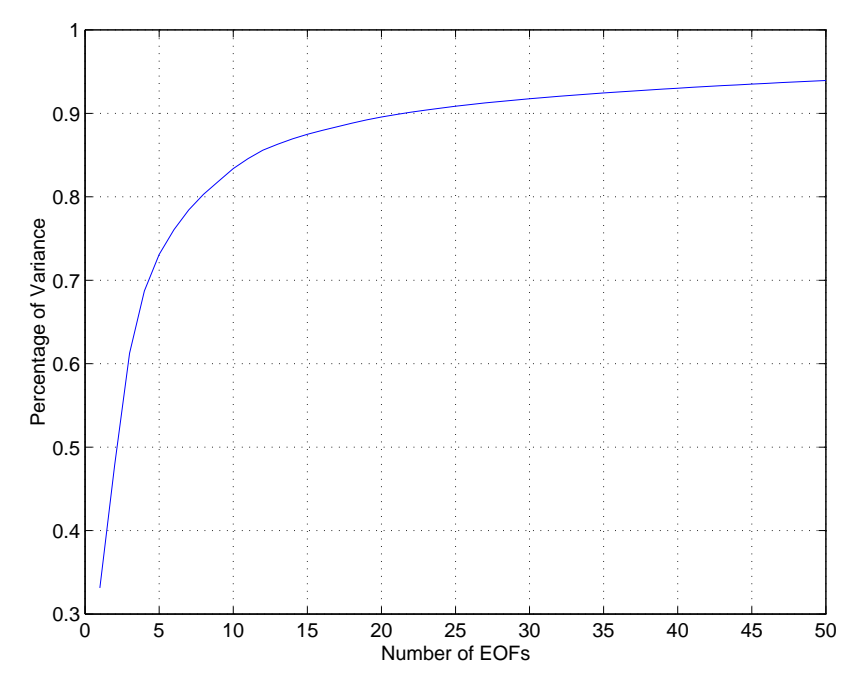

Fig. 3. Percentage of variance explained by the EOFs versus the number of retained EOFs.

where the new global correction directions $L_{k}$ evolve with the tangent linear model $\mathbf{M}$ (evaluated at $X^{f}$ ), as in the SEEK filter,

$L_{k}=\mathbf{M}\left(t_{k-1}, t_{k}\right) L_{k-1}$.

The forecast error covariance matrix can be then approximated by

$P^{f}\left(t_{k}\right)=L_{k}^{B} U_{k-1}\left(L_{k}^{B}\right)^{T}+Q_{k}$.

The structure of the forecast and analysis error covariance matrices of this filter is, therefore, very similar to that of the SEEK filter. Basically, these matrices are taken to be singular of low rank (or at least approximated to be so).

\section{Experimental setup}

The setup of the assimilation experiments is presented in this section. A general overview is given in Table 1.

\subsection{The state vector}

The variables of the physical sub-model, which are only used as forcing fields to the ecology, were considered perfect, and were therefore not included in the estimation process. This eliminates the influence of the forcing on the assimilation system performance, allowing to better understand the filter's behavior with the ecological variables. The filter's state vector contains, therefore, the 88 state variables of the ecological model only. In more realistic applications, the assumption of perfect physics can be overoptimistic and the joint estimation of physical/biological variables may be necessary for a complete control of the coupled physical-ecological system. It should be noted that some biogeochemical variables may become negative after the filter's correction. Following Natvik and Evensen (2003), these variables were simply set to a small threshold value close to zero, allowing species to become active again.

As mentioned above, the numerical grid of the Cretan Sea has 56 boxes in the zonal direction and 20 boxes in the meridional direction with 30 vertical layers. The corresponding water points for this grid are 20097, which multiplied by the 88 state variables of the ecological model, define the dimension of the state vector to be equal to 1768536 .

\subsection{Choice of the filter's correction directions}

Global and local functions were determined using the classical and local EOF analysis as described in Sect. 3.1. Following Pham et al. (1998), the EOF analysis was carried out on a set of state vectors simulated by the model itself. Therefore, the model was first integrated for 20 years with the aim of reaching a statistically steady state. Another integration of two more years was next carried out to generate a historical sequence of model realizations. A sequence $\mathcal{H}_{\mathcal{S}}$ of 360 state vectors was retained by storing one state vector every two days. As the ecological state variables are of a different nature, each variable was normalized by the inverse of its spatially-averaged (over all the grid points) standard deviation. Figure 3 plots the cumulative percentage of variance explained by the EOFs as a function of the number of EOFs. As expected, the first EOFs capture most of the variability of the system, while the last EOFs are reduced to noise. For instance, the first 20 EOFs explain $90 \%$ of the observed variability in the sample $\mathcal{H}_{\mathcal{S}}$.

Different sets of local EOFs were determined by decomposing the model domain into horizontal and vertical subdomains, according to the spatial variability of the Cretan Sea ecosystem. The local analysis was performed on the residuals of the states in the first 3, 6 and 9 global EOFs, which explain $63 \%, 72 \%$ and $80 \%$ of the total variance of the sample $\mathcal{H}_{\mathcal{S}}$, respectively. In the horizontal direction, the 

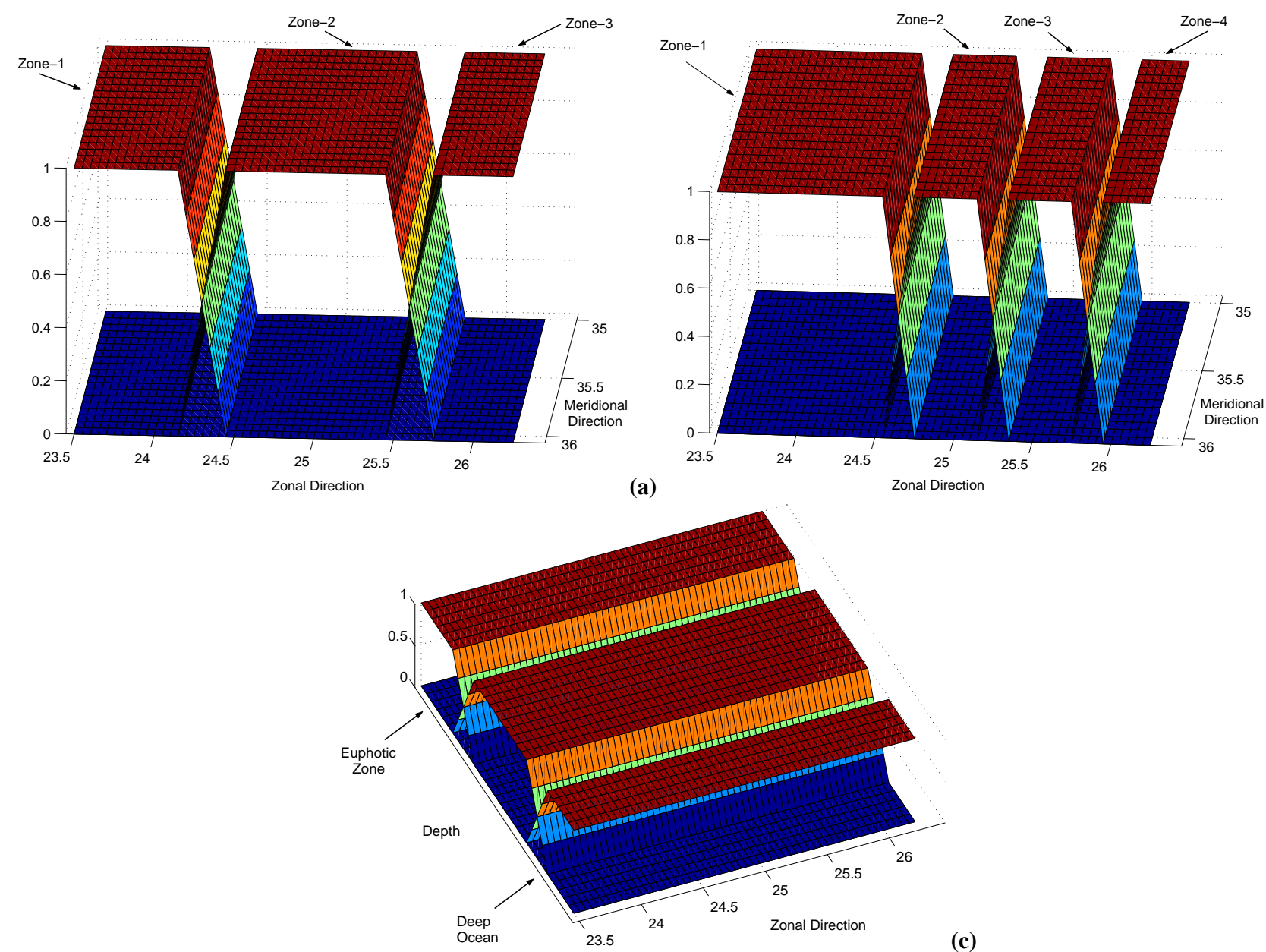

Fig. 4. Model domain decomposition into 3 and 4 horizontal sub-domains and 3 vertical sub-domains.

model domain was decomposed into three and four overlapping sub-domains, using the partitions of unity functions as shown in Fig. 4. In both cases, the width of the overlapping areas was set to $1 / 6^{\circ}$. The first decomposition was used when profile data are assimilated to isolate the observed area, as illustrated in Fig. 2, limiting in this way the range of the filter's corrections to this area only. The second decomposition was applied to isolate the different observed spatial variabilities in the model. Separate EOF analysis were then applied on the projections of the state vectors of the sample $\mathcal{H}_{\mathcal{S}}$ on each of these sub-domains. Another decomposition in the vertical direction was also carried out, according to the natural vertical variability that the ecosystem could exhibit: euphotic zone, deep-ocean, and the area in between. The depth of the euphotic sub-domain was set to the first $200 \mathrm{~m}$, with an overlapping zone of 50 meters between the two sub-domains. The deep ocean was also decomposed into two sub-domains (Fig. 4). Obviously, the local EOFs of the deep ocean zones do not produce any corrections when surface ocean color data are assimilated. The use of global functions may, therefore, be crucial in this case for an efficient propagation of surface information to the deep ocean.

\subsection{Data and filter validation - twin experiments}

A twin experiments approach was adopted for this study, in which the "truth" is assumed to be provided by the model itself. Therefore, a set of 45 reference (true) states $\left(X^{t}\right)$ is first retained from a reference model free-run, sampled every two days from March to June. This period is marked by the increased primary production of the system, and it has been chosen in order to test the effectiveness of the filters during strongly variable periods. Pseudo-observations for the assimilation experiments are then extracted from the $X^{t}$. These states are also later used to evaluate the filter's performance by comparing them with the fields produced by the assimilation system. The multivariate properties of the filters can, therefore, be assessed by examining the impact of the assimilation system on non-observed variables. Pseudoobservations of ocean color data were sampled from the reference states $X^{t}$ over the entire surface domain. Pseudoprofiles data of nitrate, phosphate, silicate and ammonia were sampled following a realistic network of 23 stations in the Cretan Sea (Fig. 2). Random Gaussian noises with zero mean and standard deviation of $5 \%$ for the profiles and $10 \%$ for the 

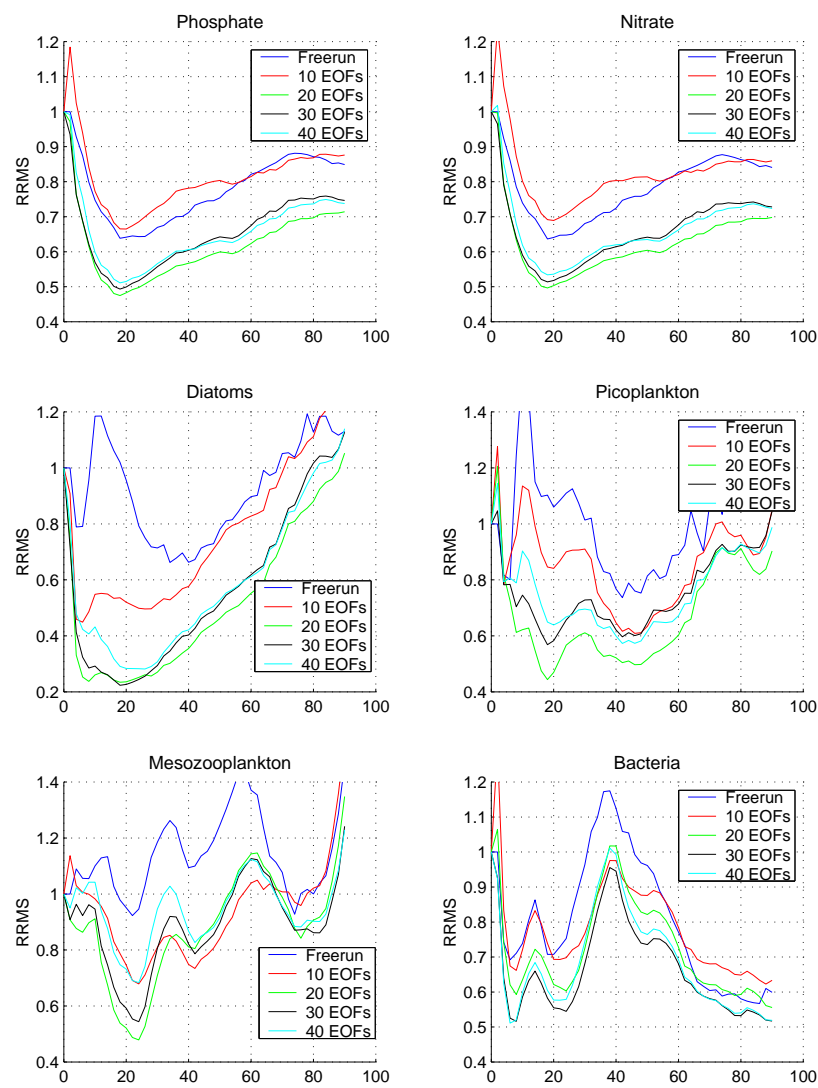

Fig. 5. Evolution in time of phosphate, nitrate, diatoms, picoplankton, mesozooplankton and bacteria RRMS from the model free-run and the SFEK filter assimilating profiles with 10, 20, 30 and 40 EOFs.

ocean color were also added to the pseudo-observations in order to construct a more realistic framework. In all the assimilation experiments, the initial state of the filter is chosen to be the mean state of the sample $\mathcal{H}_{S}$.

The performance of the filters is assessed through the relative root mean square (RRMS) error over the whole model domain for each state variable. The RRMS is defined as

$\operatorname{RRMS}\left(t_{k}\right)=\frac{\left\|X^{t}\left(t_{k}\right)-X^{a}\left(t_{k}\right)\right\|}{\left\|X^{t}\left(t_{k}\right)-\bar{X}\right\|}$,

where $X^{a}$ is the analysis state obtained by the filter, $\bar{X}$ is the mean state of the sample $\mathcal{H}_{S}$ and $\|\cdot\|$ denotes the Euclidean norm. The error is therefore relative to the free-run error, since the denominator represents the error when no observations are assimilated and the analysis vector is taken as the mean state of the historical free-run.

\section{Assimilation results}

Several experiments were carried out to evaluate the representativeness of the different set of global-local EOFs and to assess the relevance of the assimilation system. The choice of the domain decomposition to make best use of the available observations was carefully assessed. As stated before, all sensitivity experiments were performed using the SFPLEK filter since it provides an efficient low-cost way to evaluate the representativeness of the different global-local EOFs. Although the assimilation results will not be identical as if the SEPLEK filter was used, we expect the sensitivities of both filters to the different correction directions to be very similar. The experimental results are focused on those variables considered as important in revealing the dynamics of the ecosystem (nutrients, phytoplankton and bacteria), and are those parameters most often measured in sampling programs.

The forgetting factor $\rho$ is used to artificially stimulate the filter's forecast covariance matrix as a simple way to compensate for the different approximations used in the filter, as the use of low-rank covariance matrices and the linearization of the system equations. Its value is therefore a priori unknown, since it completely depends on the characteristics of the assimilation scheme and the system under study. Here we resort to experimentation to find an appropriate value for $\rho$. Several assimilation runs (not shown here) were therefore first performed with the SFPLEK filter, to study the behavior of the assimilation system with different values of $\rho$ varying between 0.5 and 1 . The best results were obtained using $\rho=0.9$, which was therefore used in all the experiments presented below.

\subsection{Assimilation of profiles of data}

The assimilation results of data profiles are first presented. The assimilation results of sea color data are discussed in the next section.

\subsubsection{Sensitivity to the number of global EOFs}

The first experiment was performed with the SFEK filter using global (classical) EOFs only. The goal was to show that the performance of the filter stagnates (even degrades in certain situations), when the number of global EOFs increases. The SFEK filter was therefore implemented with 10, 20, 30 and 40 EOFs. The performance of the filter was also compared to the model free-run (without assimilation) to assess the relevance of the assimilation system. The RRMS of these runs are illustrated in Fig. 5. The assimilation system enhances the model behavior in all cases. For most of the variables, the filter performs best with 20 EOFs. This suggests that the last global EOFs do not represent any specific mode of the system and are very often reduced to noise. Only bacteria seems to require more than 20 EOFs, but this is probably due to the short-range variations of this variable. Overall, the SFEK filter with global EOFs fails to stabilize the RRMS completely, particularly for diatoms and mesozooplankton. Assimilation results for diatoms needs to be improved and can be explained by the significant spatial and temporal variations during the winter mixing. As nutrients are transported in the euphotic zone, the conditions become particularly favorable for big cells as diatoms, significantly increasing their contribution to the overall primary production. This local 
behavior is generally difficult to capture by the global (classical) EOFs. Although mesozooplankton grows at a slower rate compared to diatoms, the latter phytoplanktonic group accounts for a significant proportion of the mesozooplankton's diet, thus explaining a part of the variability.

\subsubsection{Sensitivity to the choice of the correction directions}

The performance of the SFEK/SFPLEK filter strongly depends on the representativeness of its invariant correction basis (Hoteit et al., 2002), therefore, providing a very efficient way to measure the relevance of the different set of globallocal EOFs. Figure 6 compares the results of three experiments using 20 correction directions as follows: (i) global (classical) EOFs, 6 global and 14 local of EOFs (in each subdomain) from (ii) the horizontal and (iii) the vertical decomposition into three sub-domains, as described in Sect. 4.2. With the exception of phosphate and nitrate, one can see that the use of local EOFs clearly enhances the filter's behavior. This is consistent with the results of Hoteit et al. (2001) who found that the use of local EOFs only, may badly affect the estimation of the model variables governed by long-range circulations, such as phosphate and nitrate. The overall improvements to the fine-scale variability are still however not quite significant. We speculate that the limited impact of this choice of domain decomposition on the filter's performance is probably due to a weak spatial variability in the small domain of the model, allowing good representation of the horizontal correlations by the classical EOFs. Concerning the vertical decomposition, the separation of the euphotic zone from the deep ocean clearly improves the assimilation results. It greatly enhances the estimation of those variables with strong variability in the euphotic zone. The use of vertical EOFs seems to provide a remarkably efficient representation of the different local variabilities in the ecosystem model. For instance, the estimation of mesozooplankton follows diatoms with small error in the first 25 days of assimilation. However as spring progresses and other functional groups increase also, mesozooplankton decreases the proportion of diatoms in its diet. This, in conjunction with the high excretion rate of uptake, contributes to a strong variability and explains part of the increase in the estimation error.

\subsubsection{Sensitivity to the number of global EOFs in the global-local functions}

The aim of this experiment is to study the sensitivity of the filter to the number of global EOFs in the global-local correction basis. Once this number is fixed, the number of local EOFs to be retained in each sub-domain can then be chosen, according to the percentage of variance explained by these EOFs (as it is usually done in the classical EOFs). Two runs were performed with the SFPLEK filter, using global-local EOFs obtained from the vertical decomposition, always with 20 correction directions: (i) 6 global and 14 local, and (ii) 3 global and 17 local, respectively. The RRMS for these runs are plotted in Fig. 7. It can be seen that the use of more global
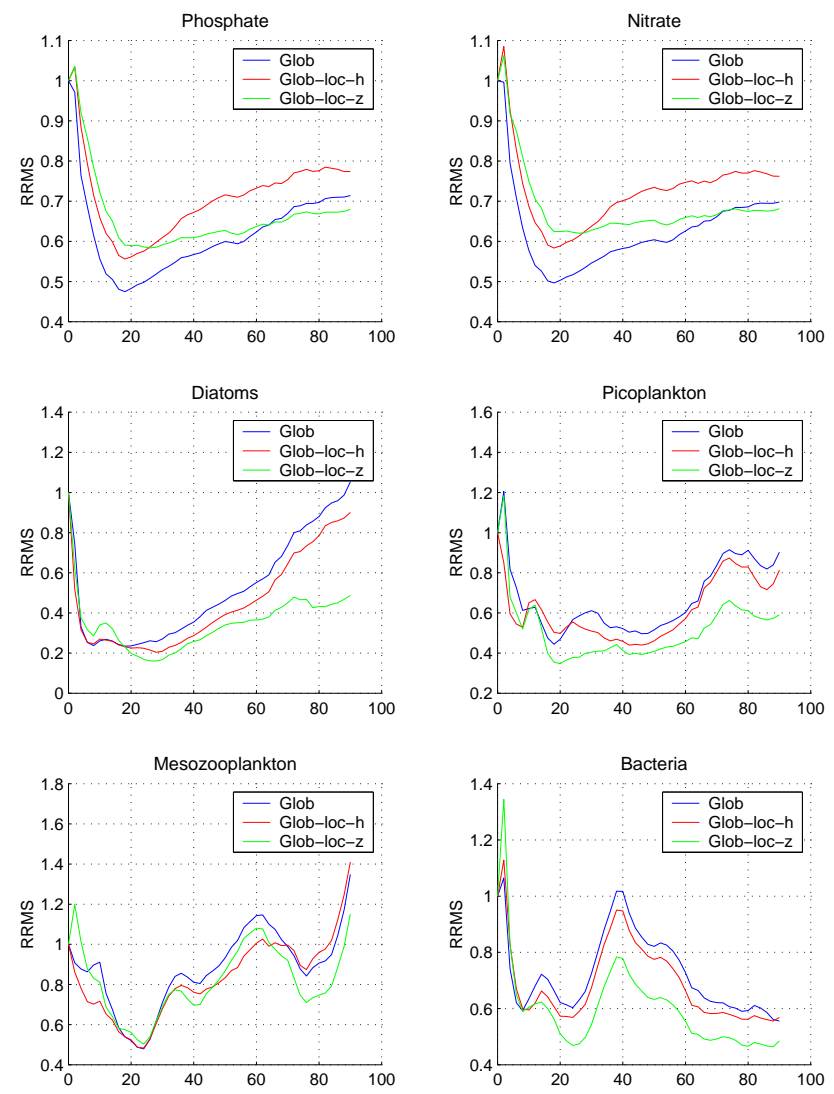

Fig. 6. Evolution in time of phosphate, nitrate, diatoms, picoplankton, mesozooplankton and bacteria RRMS from the SFEK/SFPLEK filter assimilating profiles using global EOFs (SFEK filter), globallocal EOFs (SFPLEK filter), from 3 horizontal and 3 vertical subdomains (as shown in Fig 4).

EOFs enhances the assimilation results for phosphate and nitrate. However, mesozooplankton seems to be better estimated with less global EOFs. This might be expected since nitrate and phosphate variables have a long-range variability while mesozooplankton is mostly dominated by small-scale variations. Changing the number of global EOFs has almost no effect on picoplankton and bacteria. The number of global EOFs should therefore be chosen very carefully while balancing between the estimation quality of the different variables of the model. Its choice depends only on the system under study and the variables that need to be estimated with more precision.

\subsubsection{Sensitivity to the choice of the horizontal sub- domains}

The last sensitivity experiment was carried out to study the performance of the assimilation system with respect to the choice of the horizontal sub-domains. More precisely, the SFPLEK filter was tested with two different sets of globallocal EOFs which have been computed from the two decompositions of the model domain into 3 and 4 horizontal sub-domains, as described in Sect. 4.2. One can see 

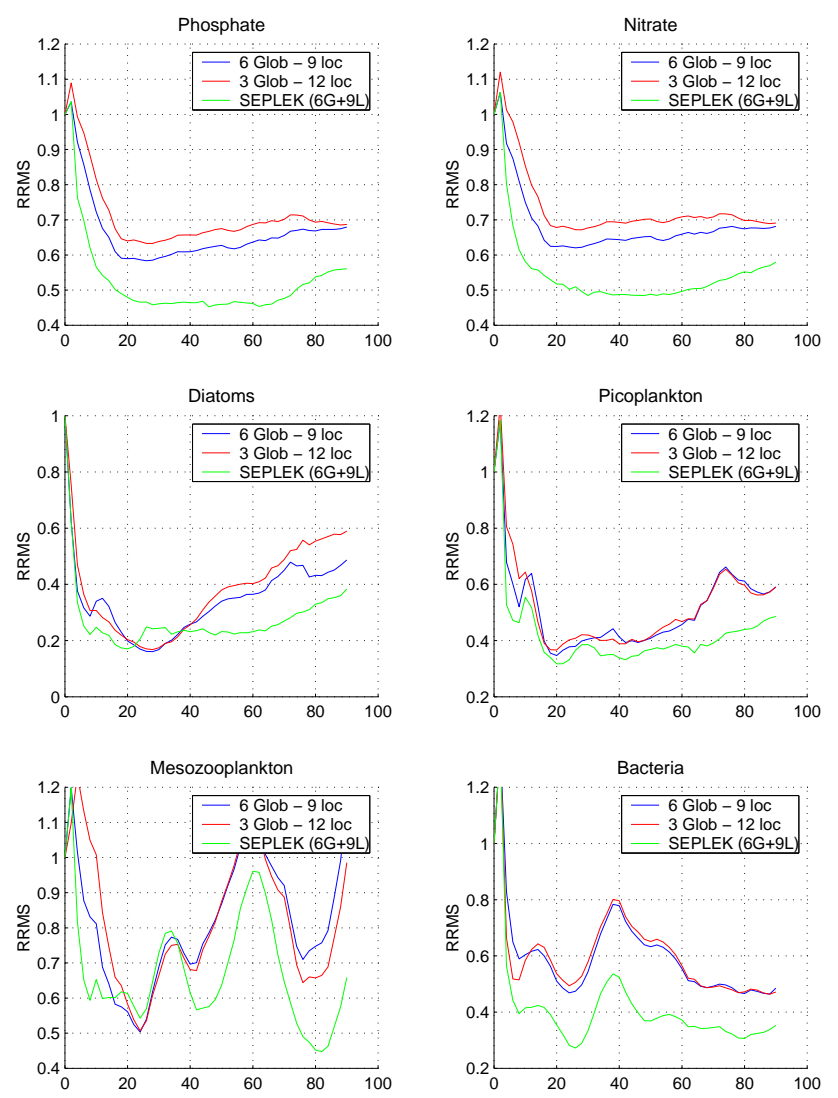

Fig. 7. Evolution in time of phosphate, nitrate, diatoms, picoplankton, mesozooplankton and bacteria RRMS from the SFPLEK filter assimilating profiles using global-local EOFs from 3 vertical subdomains with 3 global - 17 local EOFs, 6 global -14 local EOFs, and from the SEPLEK filter with 6 global - 14 local EOFs.

from the results of these runs plotted in Fig. 8 that the filter's performance highly depends on the choice of the subdomains. Moreover, increasing the number of sub-domains does not necessarily improve the assimilation results. Surprisingly, only the estimation of the picoplankton was better when more sub-domains were used. In general, limiting the length of the corrections in the horizontal direction seems to enhance the filter performance by filtering noises caused by poorly known correlations in the covariance matrix. However, the choice of the decomposition must also take into account the different local variabilities of the model, as well as the type of assimilated observations.

\subsubsection{Evolution of the global correction directions - SE- PLEK filter}

The evolution of the correction directions is important for keeping track of changes in the model dynamics. As stated in Sect. 3.4, when a set of global-local functions is used, only the global functions can be allowed to evolve in time to follow changes in the model dynamics. In this experiment, a set of 6 global and 9 vertical local EOFs (in each sub-domain) were used in the SFPLEK and the SEPLEK filters. The only
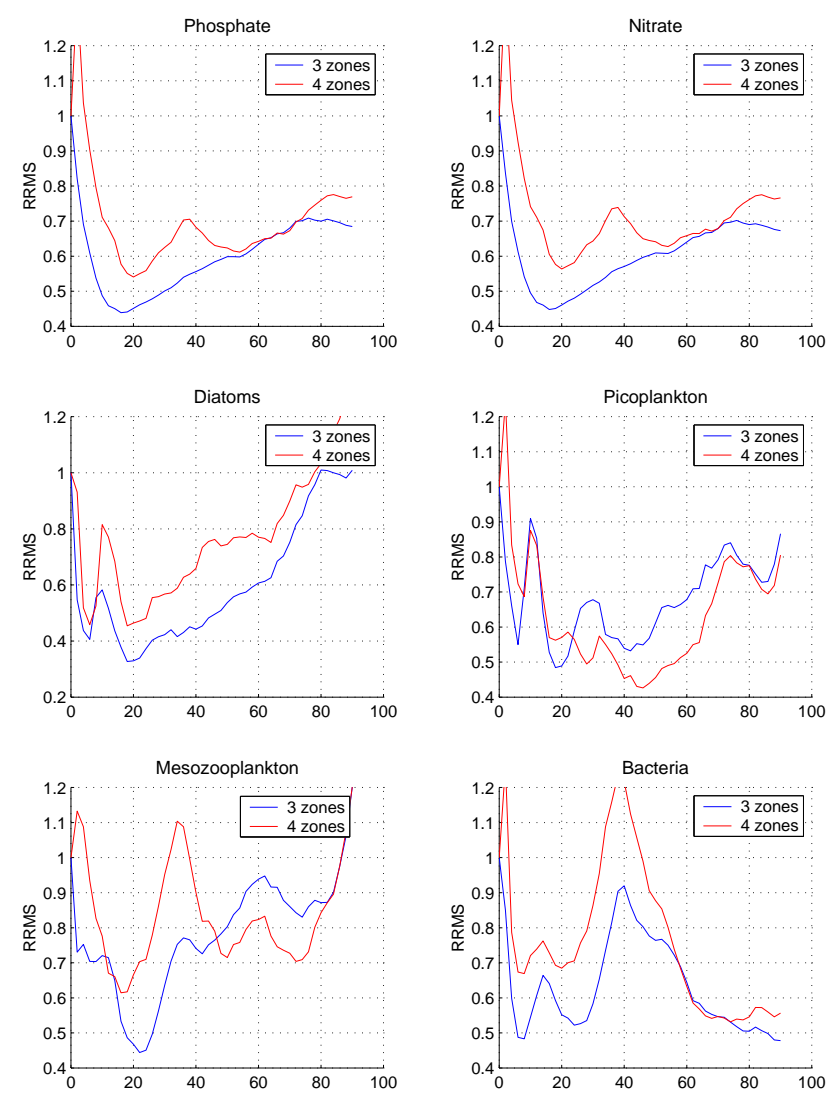

Fig. 8. Evolution in time of phosphate, nitrate, diatoms, picoplankton, mesozooplankton and bacteria RRMS from the SFPLEK filter assimilating profiles using global-local EOFs from 3 and 4 horizontal and 4 sub-domains (as shown in Fig 4).

difference between the two runs was that the global directions evolve in time in the SEPLEK filter, while they remain unchanged in the SFPLEK filter. The computational cost of the former was therefore six times higher than the latter. This is affordable with the current computers, and is noticeably faster than the SEEK filter, which usually requires the evolution of more than 30 functions for the studied ecosystem.

The higher cost of the SEPLEK filter can be supported by Fig. 7, which plots the RRMS for the two runs. Indeed, one can clearly see that the evolution of the global directions enhances the assimilation results for all the model variables. It also improves the stability of the filter due to the continuous tracking of the changes in the model dynamics. Only the RRMS for the mesozooplankton was not significantly improved. This points to difficulties in the SEEK update equation of the correction directions to follow rapid changes in the model.

Integrated chlorophyll concentrations $(0 \mathrm{~m}-120 \mathrm{~m})$ of the assimilated, the reference, and the free-runs, for the May, are plotted in Fig. 9. The filter follows closely the pseudoobservations reproducing the spatial distribution, as well as the range of values. An interesting feature is the local low concentration area at the center - top part of the domain, attributed to the existence of an intense anticyclone (Petihakis 

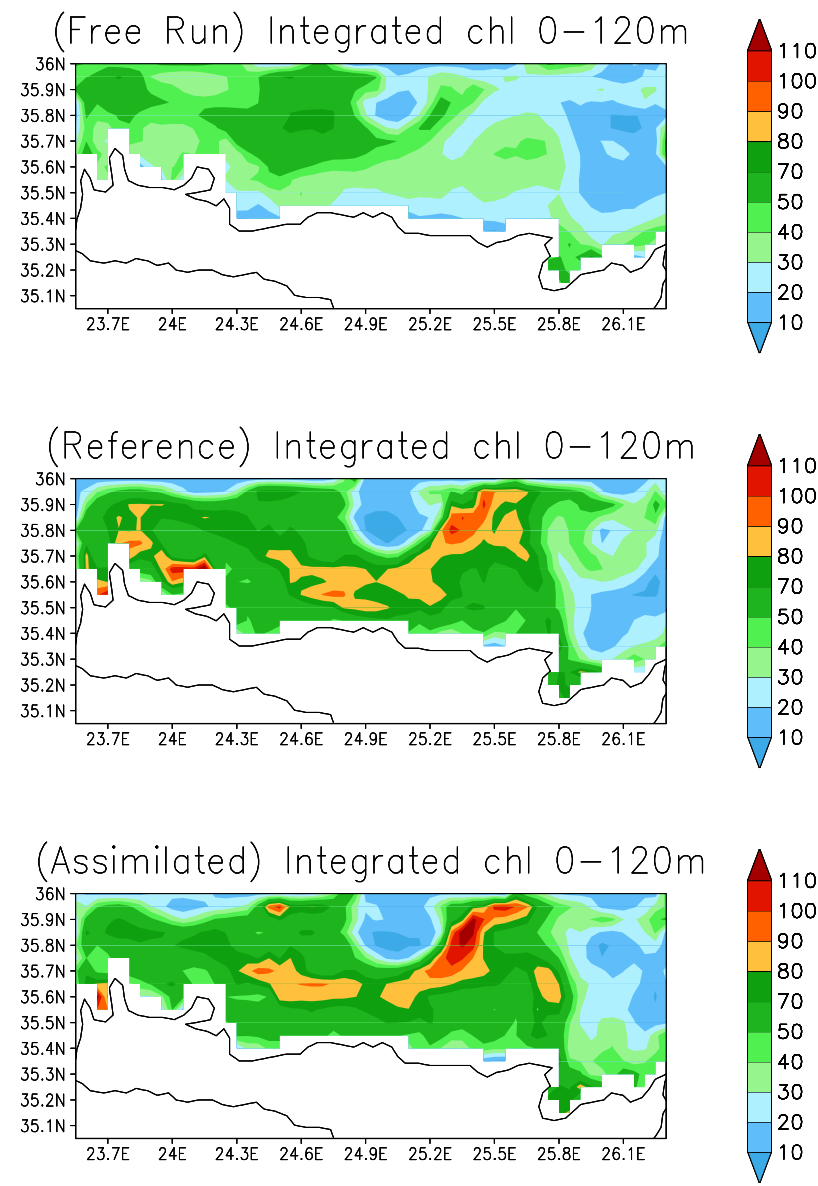

Fig. 9. Integrated chlorophyll concentrations $\left(\mathrm{mg} / \mathrm{m}^{3}\right)$ between $0 \mathrm{~m}-120 \mathrm{~m}$ from the free run, the reference run, as estimated by the SEPLEK filter.

et al., 2002; Triantafyllou et al., 2003b), transferring nutrients in the deeper layers. The action of another anticyclone is also observed at the east part of the simulation field, as is also evident by the low concentration values. Between these two gyres the intrusion of nutrients in the euphotic zone is depicted by maximum concentrations of chlorophyll. The areas with low chlorophyll concentrations, caused by the gyral system of the two anticyclones, is also nicely simulated by the free run. The later, however, fails to reproduce the increased chlorophyll concentrations close to the coast and between the two anticyclones. For the same period integrated bacterial biomass also exhibits a very good coincidence between the two runs (Fig. 10). The importance of the physical features in the ecosystem functioning is evident, with bacterial biomass following the distribution of chlorophyll, although maximum values are developed closer to the coast. This is because the spring bloom which started in the coastal areas in late winter, provides the important food substrate for bacterial growth. Additionally, a cross section of chlorophyll concentrations of the three runs (with assimilation, reference, free) along the east-west direction, at latitude $35.75^{\circ} \mathrm{N}$, is illustrated in Fig. 11. The simulations are very similar, pro-
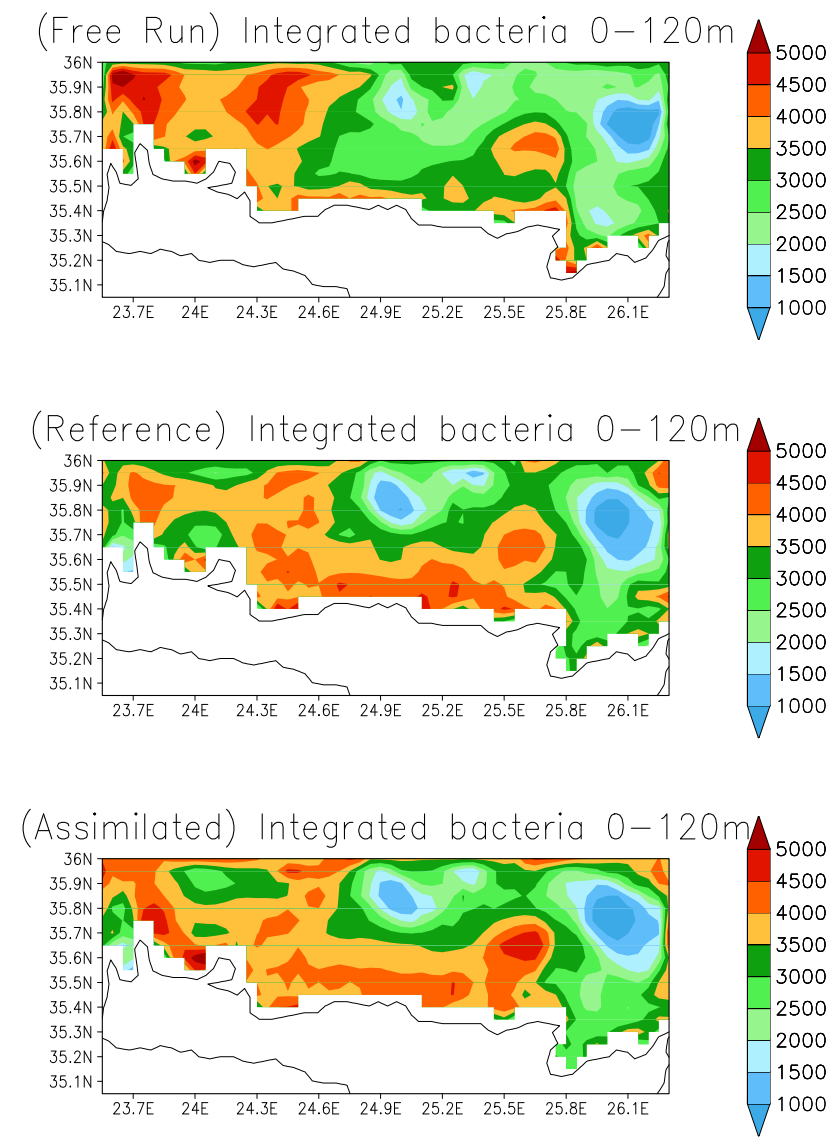

Fig. 10. Integrated bacterial biomass between $0 \mathrm{~m}-120 \mathrm{~m}$ from the free run, the reference run, as estimated by the SEPLEK filter.

ducing a characteristic feature of the Cretan Sea ecosystem, a Deep Chlorophyll Maximum (DCM), which as the thermocline formation progresses (August), reaches as deep as 90-100 m. The presence of the anticyclone at the center of the domain is nicely depicted by the low chlorophyll concentrations.

\subsection{Assimilation of ocean color data}

Assimilation experiments of ocean color data are presented in this section. The aim of these runs is to show that the choice of the domain's decomposition should take into account the characteristics of the observations to be assimilated into the model. Several sensitivity experiments using the SFPLEK filter were performed, as in the later section. Here only the assimilation results of two assimilation experiments are discussed. The first experiment deals with the sensitivity to the choice of the domain decomposition, while the second assesses the impact of the number of global EOFs in the vertical global-local functions on the filter's behavior.

For the first experiment, three runs were carried out as in Sect. 5.1.1: (i) 20 global (classical) EOFS, (ii) 6 global and 14 local EOFs for the horizontal decomposition into 4 subdomains (which provided better results in our experiments than the horizontal decomposition into 3 sub-domains), and 


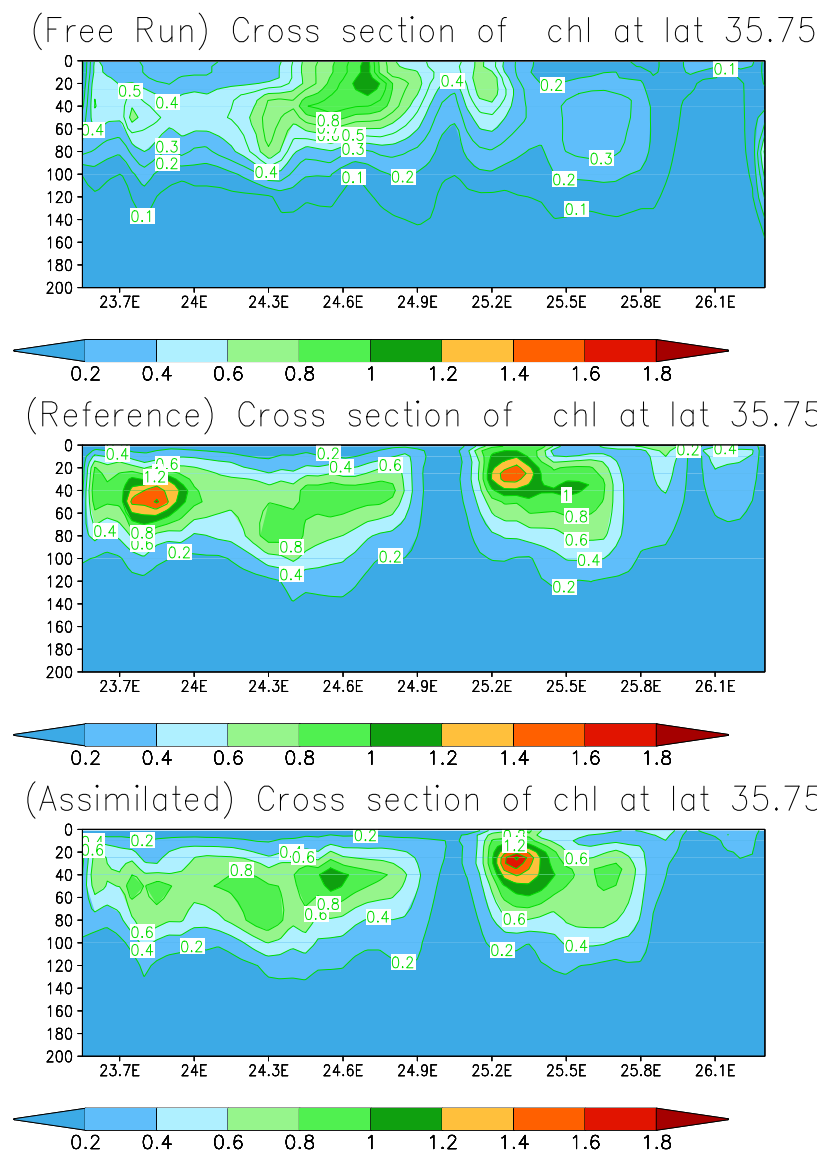

Fig. 11. Vertical cross sections of chlorophyll concentrations $\left(\mathrm{mg} / \mathrm{m}^{3}\right)$ at latitude $35.75^{\circ} \mathrm{E}$ from the free run, the reference run, as estimated by the SEPLEK filter.

(iii) 9 global and 11 local EOFs from the decomposition into vertical sub-domains. The RRMS for these runs are presented in Fig. 12. At first glance, the assimilation of sea color data significantly improves the estimation of picoplankton, mesozooplankton and bacteria compared to the experiment of assimilating data profiles. Phosphate and nitrate are well estimated by the filter, noting that these data have very little effect on diatoms. Although in the beginning of the experimental period diatoms are dependent on the availability of phosphate and nitrate (small error), soon after they are limited by silicate, while a significant pressure is also exerted by predators. Additionally, the assimilation of sea color data affects chlorophyll concentrations only at the top layer of the euphotic zone and thus it provides relatively less information about diatoms, which are mostly concentrated at deep layers because of their heavy weight. The use of global-local correction directions generally enhances the filter's behavior for all variables, although the improvements are not significant. In contrast to the assimilation of data profiles, the horizontal decomposition seems to provide better results, while the impact of the vertical decomposition is less pronounced. In particular, phosphate and nitrate are better assimilated when horizontal local EOFs are used, probably because of the global
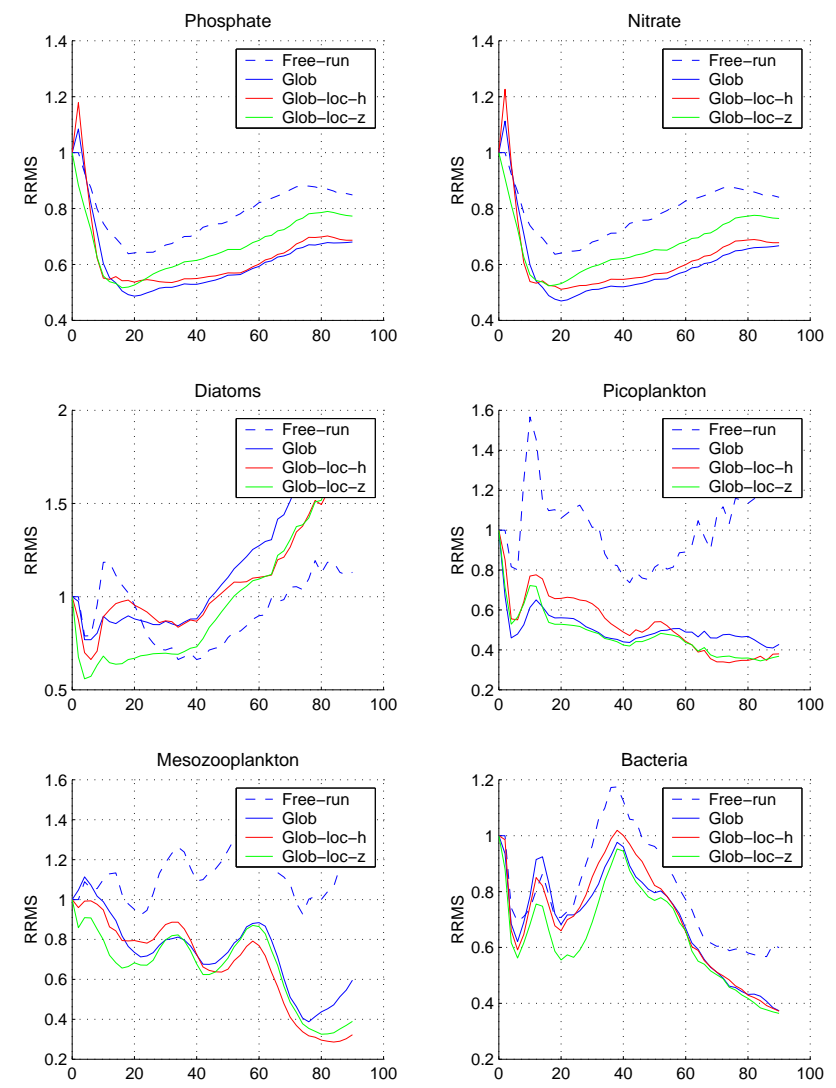

Fig. 12. Evolution in time of phosphate, nitrate, diatoms, picoplankton, mesozooplankton and bacteria RRMS from the SFPLEK filter assimilating sea color data using global EOFs, global-local EOFs from 4 horizontal and 3 vertical sub-domains (as shown in Fig 4).

coverage of color data to all sub-domains. Limiting the propagation of the surface data by using vertical local EOF, degrades the filter's performance for these two variables and as one can expect, the classical global EOFs still provide the best estimations.

In an attempt to explain why the vertical decomposition is not as efficient as in the assimilation of profiles data, three assimilation runs were performed using vertical global-local correction functions with: (i) 3 global and 17 local, (ii) 6 global and 14 local, and (iii) 9 global and 11 local. Figure 13 depicts the RRMS under the euphotic zone for the three runs. As can be seen, the use of more global EOFs, up to a certain number, improves the filter's performance in the deep ocean. This suggests that an appreciable number of global EOFs are still needed to propagate the surface data into the deep layers. This was not always true (not presented here) for all model variables in the euphotic zone, and the use of local EOFs generally improves the assimilation results. These results are not in full agreement with those of Carmillet et al. (2001), who found that the use of only local EOFs in the euphotic zone improves the filter's behavior. We speculate that this is probably due to a weak representation of the surface/subsurface correlations in their (global) EOFs. The results of this experiment, however, confirm the Carmillet et al. (2001) finding, 
namely that the existence of "bad correlations" in the correction directions has a more severe impact on the vertical than on the horizontal direction.

In another experiment similar to the one performed in Sect. 5.1.5, but assimilating ocean color data, the SEPLEK filter was used to assess the relevance of the evolution of the global correction directions. The results of this run were consistent with Sect. 5.1.5 and lead to identical conclusions. Therefore, we decided not to show them in order to save space.

\section{Summary and discussion}

While data assimilation techniques have made great progress in meteorology and physical oceanography, their application to complex ecosystem models often encounters many difficulties, mainly because of the huge number of ecological variables and of their complex variabilities. In this paper, sophisticated Kalman filters, called SFPLEK and SEPLEK, were used to assimilate profiles and sea color data into a 3D coupled biophysical model of the Cretan Sea ecosystem. These filters make use of a combination of global (classical) and so-called local EOFs to supplement the large-scale variability continued by the classical EOFs with local fine-scale information. The local EOFs are determined according to the different variability of the ecosystem model, as well as to the characteristics of the assimilated observation. Moreover, the global EOFs can be allowed to evolve with the model dynamics, and this was found to be beneficial for the stability of the filter during periods of strong ecological variability.

From the various twin experiments performed, the performances of the SFPLEK and SEPLEK filters were satisfactory for assimilating sea color data and profiles of nitrate, phosphate, silicate and ammonia from 23 stations in the Cretan Sea, without requiring large computational resources. The assimilation results also suggest that the use of local functions, obtained from a horizontal or a vertical domain decomposition, clearly improves the representativeness of the filter's correction basis. Sensitivity experiments also revealed that an efficient choice of horizontal decomposition should take into account both the different local variabilities of the system and the distribution of the data. A horizontal decomposition was found, however, to have less of an impact than a vertical decomposition, probably because of the small area of the computational field which make the classical EOFs less noisy in the horizontal direction. The assimilation experiments also confirm previous results by stating that the ecosystem models are more sensitive to bad correlations in the vertical than in the horizontal direction. The use of local vertical EOFs was found to be beneficial for enhancing the stability of the filter, as long as they are complemented with a number of global EOFs for an efficient representation of the model large-scale variability. This was more evident when sea color data were assimilated to help propagating surface information into the deep layers. More experiments with different vertical decompositions still need to be carried out
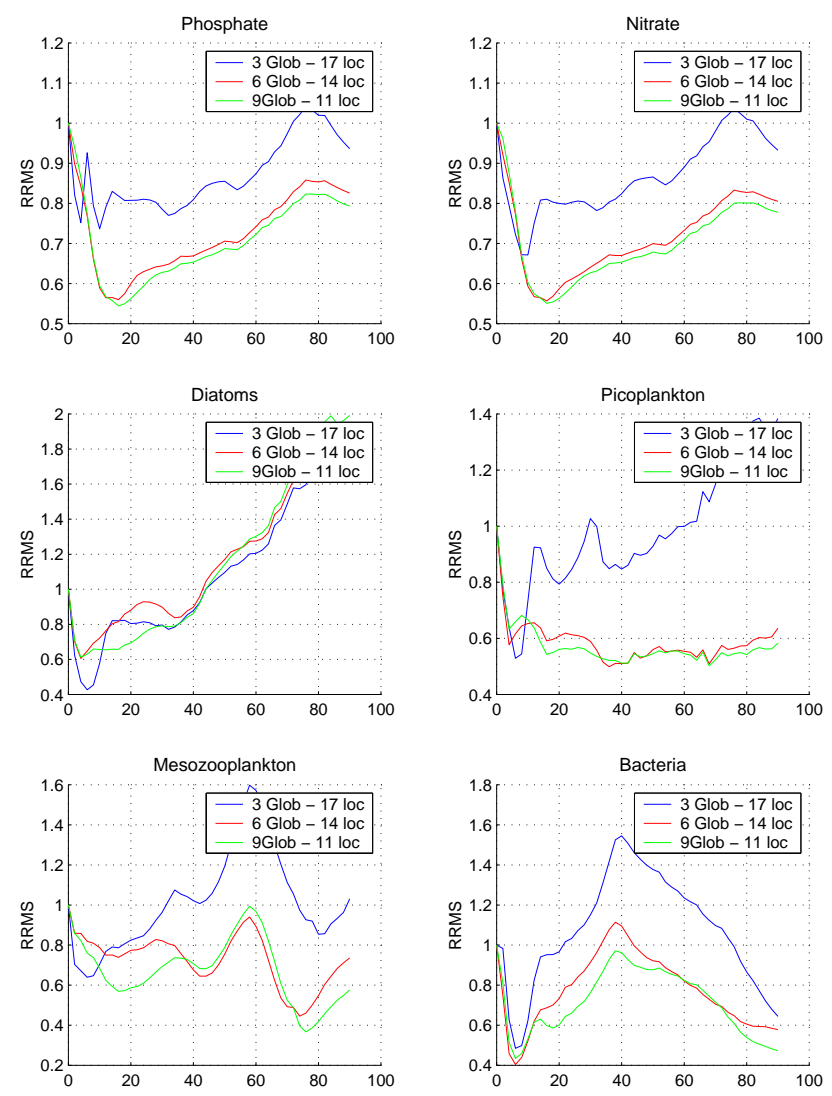

Fig. 13. Evolution in time of phosphate, nitrate, diatoms, picoplankton, mesozooplankton and bacteria RRMS in the deep ocean from the SFPLEK filter assimilating sea color data using global-local EOFs from the vertical decomposition with: (i) 6 global - 14 local EOFs, and (ii) 9 global - 11 local EOFs.

to tune the parameters of the vertical sub-domains. At this stage, however, we thought that it is more important to better understand the impact of different horizontal sub-domains, since the sensitivity of the filter to the latter is more unpredictable. Recently, the availability of ocean color data provided a unique opportunity for the problem of data assimilation in ecosystem models due to their global coverage. However, these data contain only information about the surface layers of the ocean, and therefore still need to be complemented with in-situ data of the lower layers, as suggested by the results of our experiments.

Twin experiments have demonstrated the ability of the SFPLEK and SEPLEK filters to efficiently control the evolution of the ecosystem state, while assimilating profiles and sea color data into a small area of the Cretan Sea during a period of strong ecosystem variability. As a next step, real profiles and ocean color data will be assimilated, which often proves to be much more complex and less forgiving than a twin experiments approach. This would point to the need for incorporating a more sophisticated model error, since ecosystem models are only a crude approximation of the real system under study. We also plan to test the filters using local functions obtained from simultaneous 
horizontal-vertical decompositions. The preliminary twin experiment applications reported in this paper were, however necessary, providing important knowledge and experience, and enabling the assessment of the impact of the filter on non-observed variables. An extension of the model configuration to cover the Eastern Mediterranean is a future task in the framework of the project Mediterranean Forecasting System Toward Environmental Predictions (MFSTEP) (http://www.bo.ingv.it/mfstep/), which might reveal a greater impact of a horizontal decomposition of the simulation domain.

Acknowledgements. This work was carried out within the framework of the project Mediterranean Forecasting System Toward Environmental Predictions (MFSTEP). The authors would like to thank A. Eleftheriou for his constructive criticism during the preparation of this work, and $\mathrm{M}$. Eleftheriou for her help in editing this text.

Topical Editor N. Pinardi thanks two referees for their help in evaluating this paper.

\section{References}

Allen, J., Ekenes, M., and Evensen, G.: An Ensemble Kalman Filter with a complex marine ecosystem model: Hindcasting phytoplankton in the Cretan Sea, Ann. Geophys., 21, 399-411, 2003, SRef-ID: 1432-0576/ag/2003-21-399.

Anderson, L.: A local least squares framework for ensemble filtering, Monthly Weather Review, 131, 634-642, 2003.

Baretta, J., Ebenhoh, W., and Ruardij, P.: The European Regional Seas Ecosystem Model, a complex marine ecosystem model, Netherlands Journal of Sea Research, 33, 233-246, 1995.

Bennett, A.: Inverse methods in physical oceanography, Cambridge Univ. Press, 346, 1992.

Bignami, F., Marullo, S., Santoleri, R., and Schiano, M. E.: Longwave radiation budget in the Mediterranean Sea, J. Geophys. Res., 100, 2501-2514, 1995.

Blumberg, A. F. and Mellor, G. L.: A description of a three-dimensional coastal ocean circulation model, in: ThreeDimensional Coastal Ocean Circulation Models, edited by: N. Heaps, Coastal Estuarine Science, 1-16, AGU, Washington, D.C., 4th Ed., 1987.

Brasseur, P., Ballabrera-Poy J., and Verron J.: Assimilation of altimetric observations in a primitive equation model of the Gulf stream using a singular evolutive extended Kalman filter, Journal of Marine Systems, 22(4), 269-294, 1999.

Burgers, G., Van Leeuwen, P., and Evensen, G.: Analysis scheme in the ensemble Kalman filter, Monthly Weather Review, 126, 1719-1724, 1998.

Cane, M., Kaplan, A., Miller, R., Tang, B., Hackert, E., and Busalacchi, A.: Mapping tropical Pacific sea level: data assimilation via a reduced state Kalman filter, J. Geophys. Res., 101, 22 599-22 617, 1995.

Carmillet, V., Brankart, J. M., Brasseur, P., Drange, H., Evensen, G., and Verron, J.: A singular evolutive extended Kalman filter to assimilate ocean color data in a coupled physical-biochemical model of the North Atlantic ocean, Ocean Modelling, 3, 167192, 2001.

Evans, G.: The role of local models and data sets in the Joint Ocean global Flux study, Deep Sea Research I, 46, 1369-1389, 1999.
Evensen, G. and van Leeuwen, P. J.: Assimilation of Geosat altimeter data for the Agulhas current using the ensemble Kalman filter with a quasi-geostrophic model, Monthly Weather Review, 124, 85-96, 1994.

Faugeras, B., Levy, M., Memery, L., Verron, J., Blum, J., and Charpentier, I.: Can biogeochemical fluxes be recovered from nitrate and chlorophyll data? A case study assimilating data in the Northwestern Mediterranean Sea at the JGOFS-DYFAMED station, Journal of Marine Systems, 40, 99-125, 2003.

Fukumori, I.: A partitioned Kalman filter and smoother. Monthly Weather Review, 130, 1370-1383, 2002.

Fukumori, I. and Malanotte-Rizzoli, P.: An approximate Kalman filter for ocean data assimilation: an example with an idealized Gulf Stream model, J. Geophys. Res., 100, 6777-6793, 1995.

Harmon, R. and Challenor, P.: A Markov chain Monte Carlo method for estimation assimilation into models, Ecological modelling, 101, 41-59, 1996.

Hoteit, I., Pham, D. T., and Blum, J.: A semi-evolutive partially local filter for data assimilation, Marine Pollution Bulletin, 43, 164-174, 2001.

Hoteit, I., Pham, D.-T., and Blum, J.: A simplified reduced-order kalman filtering and application to altimetric data assimilation in tropical pacific, Journal of Marine Systems, 36, 101-127, 2002.

Hoteit, I., Triantafyllou, G., Petihakis, G., and Allen, J. I.: A singular evolutive extended Kalman filter to assimilate real in situ data in a 1-D marine ecosystem model, Ann. Geophys., 21, 389-397, 2003,

SRef-ID: 1432-0576/ag/2003-21-389.

Houtekamer, P. and Mitchell, H.: Data assimilation using an ensemble Kalman filter technique, Monthly Weather Review, 126, 796-811, 1998.

Lawson, L., Hofmann, E., and Spitz, Y.: Time series sampling and data assimilation in a simple marine ecosystem model, Deep Sea Research II, 43, 625-651, 1996.

Malanotte-Rizzoli, P.: Modern approaches to data assimilation in ocean modeling, Elsevier Oceanography Series, 61, Elseiver, P.O. Box 211, $1000 A E$ Amsterdam, The Netherlands, 455, 1996.

Matear, R. J.: Parameter optimization and analysis of ecosystem models using simulated annealing: A case study at station P, Journal of Marine Research, 53, 571-607, 1995.

Natvik, L. and Evensen, G.: Assimilation of ocean color data into a biochemical model of the North Atlantic. Part-I. Data assimilation experiments, Journal of Marine Systems, 40-41, 127-153, 2003.

Natvik, L., Eknes, M., and Evensen, G.: A weak constraint inverse for a zero-dimensional marine ecosystem model, Journal of Marine Systems, 28, 19-44, 2001.

Ott, E., Hunt, B. R., Szunyogh, I., Zimin, A. V., Kostelich, E. J., Corazza, M., Kalnay, E., Patil, D. J., and Yorke, J. A.: A Local Ensemble Kalman Filter for Atmospheric Data Assimilation, Tellus, 56A, 415-428, 2004.

Petihakis, G., Triantafyllou, G., Allen, J., Hoteit, I., and Dounas, C.: Modelling the Spatial and Temporal Variability of the Cretan Sea Ecosystem, Journal of Marine Systems, 36, 173-196, 2002.

Pham, D., Verron, J., and Roubaud, M.: Singular evolutive Kalman filter with EOF initialization for data assimilation in oceanography, Journal of Marine Systems, 16, 323-340, 1998.

Testut, C., Brasseur, P., Brankart, J.-M., and Verron, J.: Assimilation of sea-surface temperature and altimetric observations during 1992-1993 into an eddy permitting primitive equation model of the North Atlantic Ocean, Journal of Marine Systems, 40-41, 
291-316, 2003.

Thingstad, T., Havskum, H., Kaas, H., Nielsen, T., Riemann, B., Lefevre, D., and Williams, P. 1. B.: Bacteria-protist interactions and organic matter degradation under P-limited conditions: Analysis of an enclosure experiment using a simple model, Limnology and Oceanography, 44, 62-79, 1999.

Triantafyllou, G., Petihakis, G., Dounas, K., and Theodorou, A.: Assessing Marine Ecosystem Response to Nutrient Inputs, Marine Pollution Bulletin, 43, 175-186, 2001.

Triantafyllou, G., Hoteit, I., and Petihakis, G.: A singular evolutive interpolated Kalman filter for efficient data assimilation in a 3D complex physical-biogeochemical model of the Cretan Sea, Journal of Marine Systems, 40-41, 213-231, 2003 a.
Triantafyllou, G., Korres, G., Petihakis, G., Pollani, A., and Lascaratos, A.: Assessing the phenomenology of the Cretan Sea shelf area using coupling modelling techniques, Ann. Geophys., 21, 237-250, 2003b,

SRef-ID: 1432-0576/ag/2003-21-237.

Verlaan, M. and Heemink, A. W.: Reduced rank square root filters for large scale data assimilation problems, Second International Symposium on Assimilation of Observations in Meteorology and Oceanograph, 1995.

Waseda, T., Jameson, L., Mitsudera, H., and Yaremchuk, M.: Optimal basis from empirical orthogonal functions and wavelet analysis for data assimilation: optimal basis WADAI, Journal of Physical Oceanography, 59, 187-200, 2003. 